\title{
67 Endurance exercise as a rehabilitation intervention after cancer
}

In the majority of patients, the malignant disease as well as the chemotherapies or radiation treatments required lead to severe impairment of physical performance and to permanent fatigue. Harmless tasks of everyday life become excruciating burdens - a state that can last for months, sometimes even years. The rapid fatigability enslaves the patient in constant rest. Exercise does not start, if at all, until many weeks after the therapy is over. That means that valuable rehabilitation time is lost.

Resting is equivalent to a lack of exercise, resulting in muscle atrophy and a further decline in performance capability.

The normal everyday activities become more and more strenuous and the need to recover grows correspondingly larger. Studies have shown, however, that carefully initiated exercise interventions are effective during and absolutely essential after cancer treatments (Brown et al 2011, Rock et al. 2012, Andersen et al. 2013, Wiskemann et Steindorf 2013). Not only are the generally positive effects of endurance training on mind and body even more beneficial for cancer patients earlier on, these patients especially profit from to the rapid boost to their immune system that is both crucial to their cure and frequently associated with a reduction in cancer-related mortality.

Carefully dosed muscle-strengthening activities involving all major muscle groups are most suitable for rehabilitation. Training programs should be designed so as to achieve approximately $70 \%$ of maximum achievable load intensity in the end. It is optimal to start with 3 half-hour training units per week, during which frequent breaks are allowed.

After 4-6 weeks, scope and intensity can be increased. Afterwards, the cancer patients may fully exert themselves during their physical activity, at least according to the results of a small, randomized, controlled study on 269 cancer patients of both sexes with a mean age of 47 years (Adamsen et al. 2009). The participants exercised nine hours per week under supervision - half the time at high intensity - and benefitted immensely from their training. Their feeling of permanent fatigue subsided markedly and vitality, emotional well-being, as well as strength and endurance improved significantly compared with a control group. By contrast, such short-term physical activity does not influence general health-related fitness and quality of life (Ballard-Barbash et al. 2012). 